\title{
Implications of the prostate intervention versus observation trial (PIVOT)
}

\author{
Timothy J Wilt \\ Asian Journal of Andrology (2012) 14, 815; doi:10.1038/aja.2012.103; Published online: 17 September 2012
}

Prostate intervention versus observation trial (PIVOT) is the largest and longest randomized trial conducted in men with early stage prostate cancer detected during the era of wide-spread prostate-specific antigen (PSA) testing. ${ }^{1}$ PIVOT enrolled 731 men and followed them for up to 15 years after randomization. PIVOT results demonstrate that compared to observation, radical prostatectomy did not result in a significant reduction in all cause or prostate cancer mortality through at least 12 years among men with localized prostate cancer; absolute differences were less than $3 \%$. Results changed little after 8 years; therefore, additional follow-up is unlikely to change our findings. Radical prostatectomy caused related harms within 30 days of surgery in $21 \%$ of men and urinary incontinence and erectile dysfunction through at least 2 years.

Prostate cancer mortality was infrequent among all men treated with observation (approximately $8 \%$ ) and rare $(3 \%)$ in men with PSA values of 10 or less or those with low-risk disease, which is important and reassuring findings for men, their families and physicians. Noncancer death rates and surgical quality in PIVOT were similar to other studies enrolling men with early stage prostate cancer. The mean age of 67 years, health status and comorbidities were similar to most men diagnosed with prostate cancer and to other trials. The effect of radical prostatectomy did not vary by patient age, race, health status or presence of other medical conditions. In men with low PSA $(\leqslant 10)$ or low-risk disease death from prostate cancer is rare in men treated with observation and surgery had no reduction in overall or prostate cancer mortality or risk of bone metastases. Surgery may reduce mortality and bone metastases in men with higher PSA or higher-risk disease. However, subgroup findings should be viewed with caution. Any positive results were based on multiple subgroups assessed, rarely met traditional levels of statistical significance, often were sensitive to methods of analysis and may be due to chance. Three-quarters of men with early stage prostate cancer have low PSA or lowrisk disease. Men diagnosed today and managed with observation likely have even a better prognosis than men enrolled in PIVOT because of detection of smaller more indolent disease. PIVOT results, combined with other data from randomized treatment and screening trials, indicate that observation should be the preferred treatment strategy for the majority of men currently diagnosed with early stage prostate cancer. Such a strategy can help men live a similar length of life, avoid death from prostate cancer and prevent treatment related harms and costs. In conclusion, PIVOT demonstrated that surgery did not reduce overall or cancer mortality compared to observation through at least 12 years' absolute differences were less than $3 \%$. While surgery may reduce mortality in the minority of men with PSA greater than 10 or with high-risk disease, it did not improve outcomes in men with PSA of 10 or less or with low-risk disease. Observation should be recommended as the preferred treatment option; it results in similar length of life prevention of prostate cancer death and avoids surgical harms. Randomized controlled trials are needed to evaluate new, more effective and safer screening and treatment approaches for men with aggressive prostate cancer.

1 Wilt TJ, Brawer MK, Jones KM, Barry MJ, Aronson WJ et al. Radical prostatectomy versus observation for localized prostate cancer. Engl J Med 2012; 367: 203-13. 